\title{
Achieving geodetic motion for LISA test masses: ground testing results
}

\author{
L. Carbone,${ }^{1}$ A. Cavalleri, ${ }^{2}$ R. Dolesi,${ }^{1}$ C. D. Hoyle,${ }^{1}$ M. Hueller, ${ }^{1}$ S. Vitale,${ }^{1}$ and W. J. Weber ${ }^{1}$ \\ ${ }^{1}$ Dipartimento di Fisica, Università di Trento, and I.N.F.N., Gruppo di Trento, 38050 Povo (TN), Italy \\ ${ }^{2}$ Centro Fisica degli Stati Aggregati, 38050 Povo (TN), Italy
}

(Dated: June 30, 2021)

\begin{abstract}
The low-frequency resolution of space-based gravitational wave observatories such as LISA (Laser Interferometry Space Antenna) hinges on the orbital purity of a free-falling reference test mass inside a satellite shield. We present here a torsion pendulum study of the forces that will disturb an orbiting test mass inside a LISA capacitive position sensor. The pendulum, with a measured torque noise floor below $10 \mathrm{fN} \mathrm{m} / \sqrt{\mathrm{Hz}}$ from 0.6 to $10 \mathrm{mHz}$, has allowed placement of an upper limit on sensor force noise contributions, measurement of the sensor electrostatic stiffness at the $5 \%$ level, and detection and compensation of stray dc electrostatic biases at the millivolt level.
\end{abstract}

PACS numbers: 04.80.Nn,07.87.+v

Among the most challenging technologies needed for the LISA gravitational wave mission is that of placing test masses in pure free fall. Accelerations due to stray forces change the distance between orbiting test masses, directly contaminating an interferometric measurement of gravitational wave strain. The LISA sensitivity goal requires acceleration noise, $a_{n}$, with spectral density below $3 \times 10^{-15} \mathrm{~m} / \mathrm{s}^{2} / \sqrt{\mathrm{Hz}}$ at frequencies down to $0.1 \mathrm{mHz}$, or force noise of order $\mathrm{fN} / \sqrt{\mathrm{Hz}}$ for a $\sim 1 \mathrm{~kg}$ test mass [1]

Environmental force noise can be screened by a satellite shield employing precision thrusters and a relative position sensor to remain centered about the free-falling test mass. The satellite, however, creates disturbances, particularly due to the close proximity of the position sensor. While noise analyses have shown proposed electrostatic sensor designs 2, 3], with 2-4 mm test mass sensor separations, to be compatible with the LISA goals, the low frequencies and extreme force isolation goals require force disturbance measurements to provide confidence in the LISA sensitivity predictions.

An ideal test of stray forces for LISA compares the differential noise in the orbits of two nearby free-falling test masses. This test will be performed, with a target acceleration noise limit of $30 \mathrm{fm} / \mathrm{s}^{2} / \sqrt{\mathrm{Hz}}$ at $1 \mathrm{mHz}$, by the LISA Test-Flight Package (LTP) [4] and Disturbance Reduction System (DRS) [5]. In preparing for such flight tests, we study the forces acting on a test mass that is nearly "free" in a single rotational degree of freedom, suspended by a thin torsion fiber inside a capacitive position sensor. The thermal torque noise limit, approached in similar apparatuses 6], is several $\mathrm{fN} \mathrm{m} / \sqrt{\mathrm{Hz}}$ at $1 \mathrm{mHz}$ for the torsion pendulum used here 7]. Dividing by half the $40 \mathrm{~mm}$ test mass width, this converts to a force noise near $100 \mathrm{fN} / \sqrt{\mathrm{Hz}}$, within a factor 100 (10) of the LISA (LTP/DRS) force noise target.

The translational acceleration noise relevant to LISA can be divided into contributions from random forces $f_{\text {str }}$ acting on the test mass (mass $m$ ) and from coupling to the relative motion of the satellite (mass $M$ ) via any dc force gradient (or "stiffness") $k_{p}$. The spacecraft motion noise arises in the position sensing noise, $x_{n}$, and in the imperfect compensation of the external forces $F_{\text {str }}$ acting on the satellite by the finite gain control loop $\left(\right.$ gain $\left.\omega_{D F}^{2}\right)$. The residual acceleration noise $a_{n}$ is

$$
a_{n}=\frac{f_{s t r}}{m}+\frac{k_{p}}{m}\left(x_{n}+\frac{F_{s t r}}{M \omega_{D F}^{2}}\right) .
$$

To characterize $a_{n}$, we use the torques, measured from the pendulum twist $\phi$, acting on a LISA-like test mass inside a realistic capacitive position sensor designed for LISA's sensing noise (spectral density $S_{x_{n}}^{1 / 2} \sim \mathrm{nm} / \sqrt{\mathrm{Hz}}$ ) and electrostatic force gradient $\left(k_{p} \sim 100 \mathrm{nN} / \mathrm{m}\right)$ requirements. The measured pendulum angular noise in the LISA measurement band establishes an upper limit on the contribution of noisy surface forces to $f_{s t r}$. Measurement of the rotational stiffness due to the AC sensing voltage characterizes a key part of the translational stiffness $k_{p}$. Finally, measurement and compensation of the sensor rotational electrostatic bias imbalance quantify and demonstrate neutralization of a potentially important contribution of stray dc electric fields to $f_{\text {str }}$.

The capacitive position sensor tested here (sketched in Fig. 1] and discussed in Refs. [8, 9]) is a variation of that projected for LTP 2, 10]. With the $40 \mathrm{~mm}$ test mass centered, the gap $d$ between all electrodes and the mass is $2 \mathrm{~mm}$. The electrodes are gold coated Mo, and are separated from the electrically grounded Mo housing by ceramic spacers. Differential gap sensing measurements from six sensing electrode pairs are combined to yield the three translational and three rotational test mass displacements. The sensor noise floor is dominated by transformer thermal noise, with $S_{x_{n}}^{1 / 2} \approx 0.3 \mathrm{~nm} / \sqrt{\mathrm{Hz}}$ and $S_{\phi_{n}}^{1 / 2} \approx 40 \mathrm{nrad} / \sqrt{\mathrm{Hz}}$. Integrated actuation circuitry can apply voltages to the sensing electrodes, used here for occasional pendulum control in $\phi$ and for electrostatic characterization of the sensor. All electrode surfaces have a dc path to a single circuit ground.

The main pendulum component is the test mass itself, a hollow gold coated Ti cube nominally $40 \mathrm{~mm}$ on a side, 


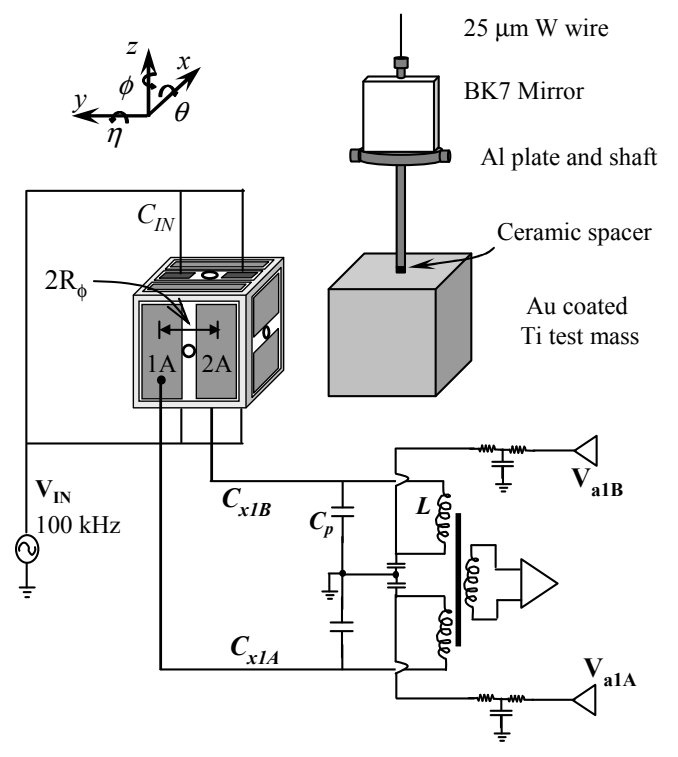

FIG. 1: Sensor electrode configuration and circuitry, with pendulum shown at right. A $100 \mathrm{kHz}$ excitation voltage is applied capacitively through four injection electrodes located on the sensor $z$ faces. Each of six sensing electrode pairs is read out by a resonant inductive bridge circuit ([8], shown here for pair \#1). The sum of the signals from pairs 1 and 2 gives the translation in $x$, and the difference, combined with the oncenter electrode separation $2 R_{\phi}=20.5 \mathrm{~mm}$, gives the rotation $\phi$ about the torsion fiber axis. Actuation voltages $\left(V_{a 1 A}\right.$ and $V_{a 1 B}$ for pair 1) can be applied to each electrode through the bridge circuitry. The nominal test mass capacitances to each injection electrode, each $x \phi$ sensing electrode, and to the entire sensor are, respectively, $C_{I N} \approx 0.6 \mathrm{pF}, C_{x} \approx 2 \mathrm{pF}$, and $C_{T} \approx 40 \mathrm{pF}$, and the excitation $V_{I N}=6 \mathrm{~V}_{r m s}$ AC biases the test mass to $\alpha V_{I N} \approx 0.4 \mathrm{~V}_{r m s}$, where $\alpha \equiv \frac{4 C_{I N}}{C_{T}}$.

with $2 \mathrm{~mm}$ walls. The test mass hangs inside the sensor, connected to the torsion fiber by an $\mathrm{Al}$ shaft passing through a hole on the top $z$ face of the sensor. The shaft supports an $\mathrm{Al}$ stopper plate, which limits the pendulum torsional range, and a silvered glass mirror, which allows an independent autocollimator readout of the pendulum torsional $(\phi)$ and swing $(\eta)$ angles. The shaft assembly is grounded through the torsion fiber and isolated from the test mass by a ceramic spacer. The total pendulum mass is $101.4 \mathrm{~g}$ (the test mass weighs $80.6 \mathrm{~g}$ ), and has a calculated moment of inertia $I=338 \pm 5 \mathrm{~g} \mathrm{~cm}^{2}$.

The torsion fiber is a nominally $25 \mu \mathrm{m} \mathrm{W}$ wire of length $1 \mathrm{~m}$. It hangs from an upper pendulum stage with an eddy current magnetic damper, which damps the pendulum swing mode with a $200 \mathrm{~s}$ decay time. The pendulum and sensor are mounted on independent micropositioners, allowing six degree of freedom adjustment of the relative position of test mass inside the electrode housing. The apparatus vacuum chamber is evacuated below $10^{-5} \mathrm{mBar}$ and sits in a thermally controlled room with $50 \mathrm{mK}$ long term stability.

With the sensor excitation on, the free torsional oscilla-

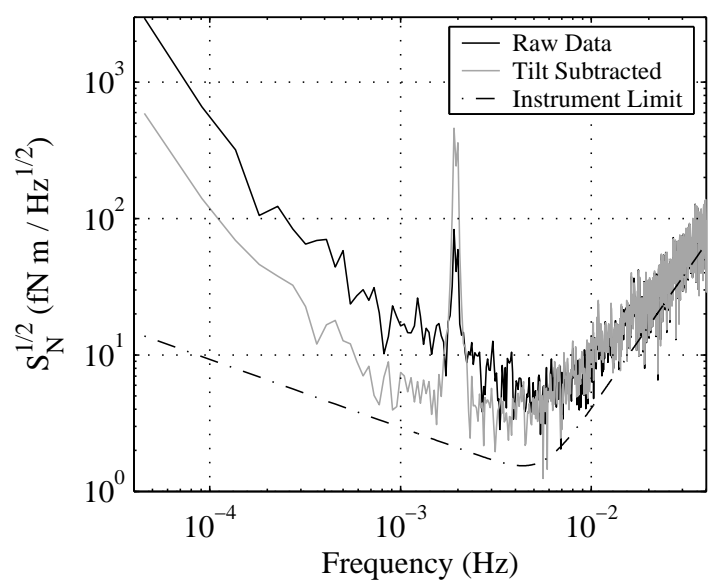

FIG. 2: Plot showing the raw (dark) and tilt subtracted (light) pendulum torque noise, with the instrument limit (dashed). Spectra for this $19 \mathrm{~h}$ measurement are calculated with a 22,000 second Hanning window, which leaves an artificial peak near the $2 \mathrm{mHz}$ pendulum resonance.

tion period $T_{0}$ is 515.1 seconds, with a quality factor $Q \approx$ 1700. The period falls to 510.3 seconds with the sensor bias off (the period change is due to a negative torsional sensor stiffness, to be discussed shortly). These pendulum dynamics, combined with the calculated moment of inertia, allow conversion of measured angular deflections in $\phi$ into torques, using the transfer function

$$
N(\omega)=\phi(\omega) \times I \omega_{0}^{2}\left[1-\left(\frac{\omega}{\omega_{0}}\right)^{2}+\frac{i}{Q}\right],
$$

where $\omega_{0}=\frac{2 \pi}{T_{0}}$ and we assume a frequency independent pendulum loss angle $\frac{1}{Q}$.

Figure 2 shows typical torque noise data, calculated from the pendulum angular noise using Eqn. 2, Also shown is the instrument limit given by the quadrature sum of the pendulum thermal noise, $S_{N, t h}^{1 / 2}=\sqrt{4 k_{B} T \frac{I \omega_{0}^{2}}{\omega Q}}$, and the sensor readout noise, $S_{\phi_{n}}^{1 / 2}$, converted into torque noise, which dominates above $5 \mathrm{mHz}$. Near $1 \mathrm{mHz}$, the raw torque noise is roughly 10 times the thermal limit. We found the main excess noise source to be a coupling to translational motion between the sensor and pendulum, due to laboratory tilt noise. This coupling, with magnitude of order $10^{-7} \mathrm{Nm} / \mathrm{m}$, is considerably stronger than reported torsion fiber "tilt - twist" couplings [] $[$ and is likely related to an electrostatic interaction involving the dielectric mirror edges. To better characterize the sensor contribution to $f_{\text {str }}$, in the absence of this coupling to lab motion, we calculate the instantaneous coupling torque and subtract the resultant twist from the $\phi$ time series. The tilt, measured in the $x$ and $y$ sensor readouts, is converted to torque through the measured "tilt-twist" coupling coefficients and then to angular twist through the pendulum transfer function (Eqn. 2). The remain- 


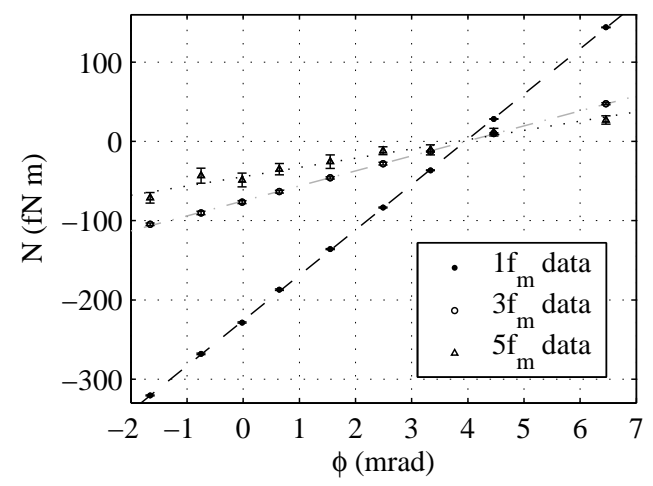

FIG. 3: $1 f_{m}, 3 f_{m}$, and $5 f_{m}(5,15$, and $25 \mathrm{mHz})$ torque component data for the squarewave modulated stiffness measurement. The roughly 5:3:1 ratio of the slopes corresponds to the $1 / f$ squarewave spectral content. The sub-fN $m$ resolution for the $1 f_{m}$ data is smaller than the point markers.

ing torque noise is roughly twice the pendulum thermal noise at millihertz frequencies and below $10 \mathrm{fN} \mathrm{m} / \sqrt{\mathrm{Hz}}$ from 0.6 to $10 \mathrm{mHz}$. The excess low frequency noise is highly correlated with the temperature fluctuations, but, to avoid subtracting a possible sensor-related temperature effect, no further correction has been made.

The low noise pendulum allows precise measurement of coherently modulated torque disturbances; we present here two such measurements. The first concerns the electrostatic stiffness 8, 9, 10] associated with the $100 \mathrm{kHz}$ sensor excitation voltage. In the approximation that all electrode surfaces $i$ are grounded, directly or by the readout circuitry, the test mass feels a negative electrostatic spring proportional to the square of the bias amplitude, in rotation as well as translation. Considering only the dc average $\left\langle V_{I N}^{2}\right\rangle$, ignoring the torque at $2 \times 100 \mathrm{kHz}$, the stiffness torque is given by

$$
N=\frac{\alpha^{2}\left\langle V_{I N}^{2}\right\rangle}{2}\left(\phi-\phi_{0}\right) \sum_{i}\left(\frac{\partial^{2} C_{i}}{\partial \phi^{2}}\right) \equiv-\Gamma_{s}\left(\phi-\phi_{0}\right)
$$

Here $\phi_{0}$ is the (unstable) electrostatic equilibrium angle where this torque vanishes.

To measure the sensing stiffness $\Gamma_{s}$, we modulate the effect by switching the $6 \mathrm{~V}_{R M S}$ injection voltage on and off at frequency $f_{m}=5 \mathrm{mHz}$. The resultant pendulum deflection, measured by the autocollimator, is proportional to $\Gamma_{s}$, and the amplitudes at odd multiples of $f_{m}$ reflect the $1 / f$ dependence of the squarewave torque's Fourier coefficients. The measurement is made over a range of test mass angles $\phi$ by rotating the pendulum suspension point. Linear fitting of torque amplitude versus $\phi$ for each odd harmonic of $f_{m}$ gives estimates of $\Gamma_{s}$ and $\phi_{0}$ (the latter measured relative to the sensor zero).

Figure 3 shows modulated stiffness torques for the first three odd harmonics of $f_{m}$. The resulting estimates for $\Gamma_{s}$ and $\phi_{0}$, shown with their statistical uncertainties in Table \are in agreement for the three harmonics.
TABLE I: Electrostatic stiffness measurement results

\begin{tabular}{ccc}
\hline \hline Component & $\Gamma_{s}(\mathrm{fN} \mathrm{m} / \mathrm{mrad})$ & $\phi_{0}(\mathrm{mrad})$ \\
$1 f_{m}$ & $-89.76 \pm 0.11$ & $3.95 \pm 0.01$ \\
$3 f_{m}$ & $-89.3 \pm 0.7$ & $3.97 \pm 0.07$ \\
$5 f_{m}$ & $-92 \pm 6$ & $3.8 \pm 0.4$ \\
\hline \hline
\end{tabular}

Though the statistical resolution for $\Gamma_{s}$ (for $\left.1 f_{m}\right)$ in a given measurement is below $1 \%$, repeated measurements have yielded a scatter of $\pm 5 \%$, which is currently being investigated. The measurement has also been performed at a fixed angle for a range of $V_{I N}$, verifying the expected $\Gamma_{s} \propto\left\langle V_{I N}^{2}\right\rangle$ dependence. The range of $\Gamma_{s}$ measured with the modulation technique is consistent with the observed period change for sensor bias on and off, which yield $\Gamma_{s}=-96 \pm 3 \mathrm{fNm} / \mathrm{mrad}$. A prediction for $\Gamma_{s}$ obtained using a finite element capacitance calculation 11] gives $-96 \pm 5 \mathrm{fN} \mathrm{m} / \mathrm{mrad}$, with the uncertainty dominated by machining tolerances. The measured several mrad offsets between the torque and sensor zeros, which would coincide in a geometrically perfect sensor, are also consistent with the machining tolerances.

Another potentially important noise source for LISA is stray dc electrostatic fields, associated with patch or surface contamination effects. A charged test mass feels force (torque) proportional to the net linear (rotational) imbalances in the electrostatic potential on the surrounding sensor surfaces. For the cosmic ray charging expected for LISA [12], net dc potential imbalances of order $10 \mathrm{mV}$ can produce significant low frequency acceleration noise [10, 13]. To measure dc imbalances, we simulate a charge modulation by biasing the mass with a voltage $V_{\Delta} \sin 2 \pi f_{m} t$ applied to the injection electrodes and measure the resulting pendulum torque 14 .

Assigning a mean stray dc voltage $\delta V_{i}$ to each conductor, the $1 f_{m}$ torque produced in this measurement is

$$
N_{1 f_{m}}=-\alpha V_{\Delta} \sin 2 \pi f_{m} t \times \sum_{i}\left(\frac{\partial C_{i}}{\partial \phi}\right) \delta V_{i}
$$

In principle, to reflect the electrostatic potential nonuniformity, the sum over electrodes $i$ should be an integral over all surface domains with different potentials. In the naive but useful model where each electrode has a spatially uniform potential, the sum in Eqn. 4 reduces to $C_{x}\left(R_{\phi} / d\right) \Delta_{\phi}$, where $\Delta_{\phi}$ is the rotational dc imbalance in the four $x \phi$ electrodes (see Fig. 4) and we assume an infinite plate model for the capacitance derivatives.

The torque in Eqn. 4, and likewise the associated random charge disturbance, is proportional to a net rotational dc bias imbalance, which can be compensated by counter-biasing the $x \phi$ sensing electrodes with the actuation circuitry. Figure 4 shows measured $1 f_{m}$ torques as a function of the compensation voltage $V_{C}$. The measured torque is nulled very close to $V_{C}=39 \mathrm{mV}$ (thus 

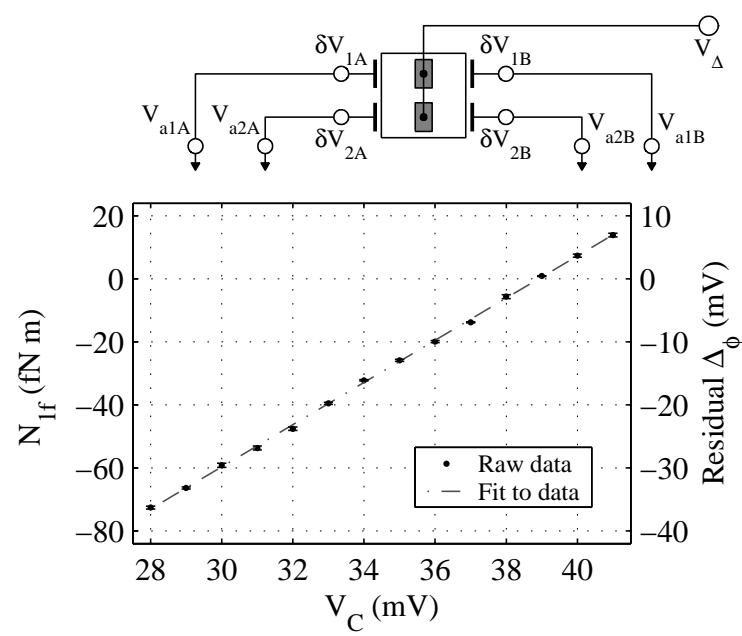

FIG. 4: Plot of dc bias measurement torques for applied bias amplitude $V_{\Delta}=3 \mathrm{~V}$ at $f_{m}=5 \mathrm{mHz}$, as a function of the applied dc compensation voltage $V_{C}$. The cartoon illustrates the measurement scheme, the average stray dc biases (with net rotational imbalance $\left.\Delta_{\phi} \equiv \delta V_{1 A}+\delta V_{2 B}-\delta V_{1 B}-\delta V_{2 A}\right)$ and the applied compensation voltages (here, we set $V_{a 1 A}=$ $\left.V_{a 2 B}=-V_{a 1 B}=-V_{a 2 A}=V_{C}\right)$. The measured torque amplitude is proportional to a residual dc imbalance, which, for spatially uniform stray dc biases, is $\Delta_{\phi}+4 V_{C}$.

$\Delta_{\phi} \approx-160 \mathrm{mV}$ ), with balancing of the residual $\Delta_{\phi}$ possible within $1 \mathrm{mV}$. An additional long measurement made for $V_{C}=39 \mathrm{mV}$ has shown millivolt level stability in the residual imbalance over a $50 \mathrm{~h}$ period.

The results presented here, derived from measurements of torque on a suspended hollow test mass that is free only along a single rotational axis, merit some discussion concerning their representativity of the translational forces acting on an orbiting, solid Au/Pt LISA test mass. For the rotational stiffness measurement, $\Gamma_{s}$ is proportional to the translational sensing stiffness $k_{s}$, with $\partial^{2} C / \partial \phi^{2}$ replaced by $\partial^{2} C / \partial x^{2}$ in Eqn. 3 The sensor ac bias is likely the dominant stiffness source, but it is not the only one. The apparatus is being upgraded to measure the full sensor-test mass coupling by modulating the sensor rotation in $\phi$. The measurements here confirm the sensor electrostatic model and convert to a translational stiffness uncertainty of $15 \mathrm{nN} / \mathrm{m}$, roughly $5 \%$ of the total LISA stiffness budget [4].

The rotational dc bias measurement and compensation shown here is also directly applicable to the translational dc bias imbalance of relevance to LISA acceleration noise. Modulating $V_{\Delta}$ also excites a coherent force, detectable in flight 14], proportional to the translational imbalance, $\Delta_{x}$. The uniform potential model for $\Delta_{\phi}$ is valid only as a rough number, as it neglects spatial surface potential variation. However, the measurement itself is sensitive to a sum over all surface domains, and thus the compensation $V_{C}$ that nulls the modulated torque (or force) will also null the torque (or force) produced by test mass charging. Compensating a $100 \mathrm{mV}$-level imbalance to within $1 \mathrm{mV}$ reduces the potentially important random charging effect to an insignificant acceleration noise level.

The torque noise data can be cautiously converted into upper limits on specific contributions to the force noise $f_{n}$ acting on LISA test masses. The hollow test mass used here is largely immune to gravitational or magnetic fields coupling to the bulk LISA test masses, and the torsional mode is insensitive to net forces from important linear temperature or field gradient effects [2]. This pendulum is designed for maximum sensitivity to surface forces, arguably the most dangerous and unpredictable sources for sensors with several-mm gaps. Any electrostatic interaction between $100 \mathrm{kHz}$ circuit noise and the sensor excitation, or between dc biases and low frequency voltage noise, produces torque noise proportional to the net force noise. For these effects, the appropriate arm length for converting to force noise is the electrode halfseparation $R_{\phi}=10.25 \mathrm{~mm}$, which gives a force noise upper limit of $1 \mathrm{pN} / \sqrt{\mathrm{Hz}}$ between 0.6 and $10 \mathrm{mHz}$ for such sources. Molecular impacts give force and torque noise on all test mass faces and have a $20 \mathrm{~mm}$ effective arm length, yielding a $500 \mathrm{fN} / \sqrt{\mathrm{Hz}}$ upper limit from 0.6 $10 \mathrm{mHz}$, with $250 \mathrm{fN} / \sqrt{\mathrm{Hz}}$ around $3 \mathrm{mHz}$. This last number corresponds, for a solid $\mathrm{Au} / \mathrm{Pt}$ test mass of the same dimensions, to acceleration noise of $200 \mathrm{fm} / \mathrm{s}^{2} / \sqrt{\mathrm{Hz}}$, a factor 7 above the LTP flight goal.

We thank E. Adelberger and B. Hamilton for reviewing this manuscript and D. Shaul for electrostatic analysis. This work was supported by ESA, INFN, and ASI.

[1] P. Bender et al, LISA ESA-SCI(2000)11, 2000.

[2] R. Dolesi et al., Class. Quant. Grav. 20, S99 (2003).

[3] B. Schumaker, Class. Quant. Grav. 20, S239 (2003).

[4] D. Bortoluzzi et al., Class. Quant. Grav. 20, S89 (2003).

[5] J. Hanson et al., Class. Quant. Grav. 20, S109 (2003).

[6] G. L. Smith et al., Phys. Rev. D 61, 022001 (1999).

[7] M. Hueller et al., Class. Quant. Grav. 19, 1757 (2002).

[8] A. Cavalleri et al., Class. Quant. Grav. 18, 4133 (2001).

[9] W. J. Weber et al., Class. Quant. Grav. 19, 1751 (2002).

[10] W. J. Weber et al., SPIE Proc. 4856, 31 (2002).

[11] D. N. A. Shaul and T. J. Sumner, SPIE Proc. 4856, 43 (2002).

[12] H. M. Araùjo, A. Howard, D. N. A. Shaul, and T. J. Sumner, Class. Quant. Grav. 20, S311 (2003).

[13] P. L. Bender, Class. Quant. Grav. 20, S305 (2003).

[14] W. J. Weber et al., gr-qc/0309067 (to be published). 